\title{
Characterization of Pulsed High-Current Generator for Extreme Ultraviolet Generation
}

\author{
Nobuaki Oshima Student Member (EDI, Nagaoka Univ. of Tech.,n_oshima@etigo.nagaokaut.ac.jp) \\ Syouichi Inokuchi Student Member (EDI, Nagaoka Univ. of Tech., inokuchi@etigo.nagaokaut.ac.jp) \\ Toru Kimura Non-member (EDI, Nagaoka Univ. of Tech., t_kimura@etigo.nagaokaut.ac.jp) \\ Weihua Jiang Member (EDI, Nagaoka Univ. of Tech., jiang@vos.nagaokaut.ac.jp) \\ Kiyoshi Yatsui Member (EDI, Nagaoka Univ. of Tech., yatsui@vos.nagaokaut.ac.jp) \\ Akira Tokuchi Non-member (Nichicon Corp., tokuchi@nichicon.co.jp)
}

Keywords : pulsed power, EUV, discharge produced plasma, magnetic pulse compression

In recent years, extensive efforts have been made toward development of extreme ultraviolet (EUV) radiation sources for applications in semiconductor lithography. EUV radiation can be obtained from high-density and high-temperature plasma which can be generated by either high-energy pulsed laser or high-current pulsed discharge. We have studied the characteristics of the high-current generator with emphasis on the electrical efficiency and the impedance matching with the discharge load, where special efforts have been made in voltage and current diagnostics.

Figure 1 shows (a) the waveforms of load voltage and current, (b) waveforms of visible and EUV light emission obtained by photodiodes, and (c) load impedance and energy obtained from the waveforms of (a). There is an unavoidable distance between the measurement point of load voltage and the capillary. Although the inductance $\left(\mathrm{L}_{\mathrm{D}}\right)$ caused by this distance is very small (on the order of $\mathrm{nH}$ ), its effect is enhanced by the large current rising-rate (on the order of $10^{11} \mathrm{~A} / \mathrm{S}$ ). The value of the inductance is approximately $10 \mathrm{nH}$, which is determined by measuring the voltage and current when the capillary is shorted by a metal rod. Therefore, the voltage waveform shown in Fig. 1(a) is obtained by subtracting $\mathrm{L}_{\mathrm{D}} \mathrm{di} / \mathrm{dt}$ from the measured voltage waveform. However, it has to be noted that, although the inductance correction described above is carried out with enough care, the dynamic variation of the load inductance is not taken into account. From Fig. 1(b), it is seen that maximum EUV radiation occurs around the time of peak discharge current which, according to a visible light signal. In fact, the maximum EUV radiation is obtained after optimization by adjusting the charging voltage and the gas pressure. The results obtained above have given us information about the performance of the high-current generator with a dynamic load of capillary discharge. The circuit behavior of the last stage of the magnetic pulse compression has been clarified and the time-resolved movement of the discharge plasma has been observed. The electrical response of the output waveforms has

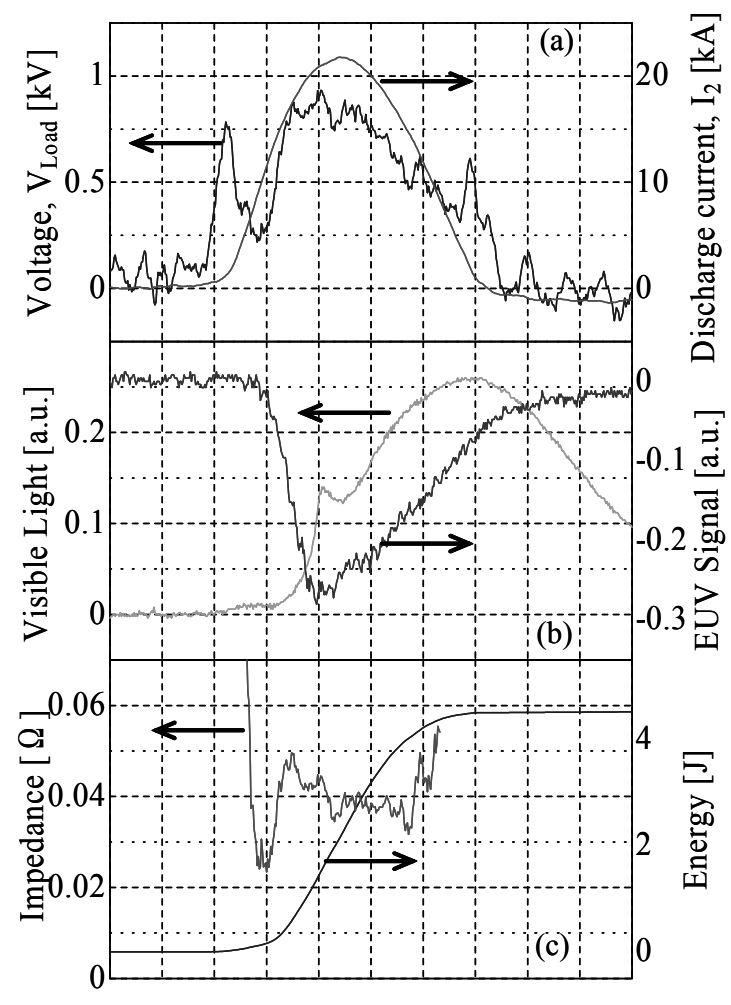

Time $100 \mathrm{~ns} / \mathrm{div}$

Fig. 1. (a) Waveforms of load voltage and current, (b) Waveforms of visible and EUV light emission, (c) Load impedance and energy

been analyzed by using diagnostic results and the overall energy efficiency and electrical resistance during the discharge have been obtained from the analytic results. As a result, the total energy deposited in the plasma per pulse was obtained to be $\sim 4.5 \mathrm{~J}$, among which only $\sim 1.4 \mathrm{~J}$ was deposited before the pinch occurred. 


\title{
Characterization of Pulsed High-Current Generator for Extreme Ultraviolet Generation
}

\author{
Nobuaki Oshima* Student Member \\ Syouichi Inokuchi* ${ }^{*}$ Student Member \\ Toru Kimura* Non-member \\ Weihua Jiang* Member \\ Kiyoshi Yatsui* Member \\ Akira Tokuchi** Non-member
}

The characteristics of a pulsed high-current generator has been studied for applications in extreme ultraviolet (EUV) generation. The objective is to clarify the electrical response of the generator circuit to the dynamic behavior of the discharge load. The experiments were carried out by driving a capillary discharge, where the plasma behavior is monitored by using high-speed camera and photodiodes. The generator performance is monitored by voltage and current probes. From the diagnostic results, the behavior of the last magnetic switch has become clear and the electrical efficiency of the generator system is obtained. The energy deposited in the capillary is calculated to be $4.5 \mathrm{~J}$ and the temporary plasma impedance at the peak current is observed to be $40 \mathrm{~m} \Omega$.

Keywords : pulsed power, EUV source, discharge produced plasma, capillary discharge, magnetic pulse compression

\section{Introduction}

In recent years, extensive efforts have been made toward development of extreme ultraviolet (EUV) radiation sources for applications in semiconductor lithography ${ }^{(1)-(3)}$. The background is the continuous miniaturization of the elementary scales in integrated circuits, driven by the pursuit of chip function and capacity. Since the resolution of optical lithography depends very much on the wavelength, the light sources employed by lithography has been evolving in the last decades from mercury lamps to different types of excimer lasers. Now, as the era of excimer laser approaches its final moment, the developers' eyes are directed onto the EUV wavelength near $13.5 \mathrm{~nm}$, which is expected to be the light source for next-generation lithography ${ }^{(4)}$.

EUV radiation can be obtained from high-density and high-temperature plasma which can be generated by either high-energy pulsed laser ${ }^{(5)(6)}$ or high-current pulsed discharge ${ }^{(7)}$. Both approaches have been studied intensively by many research and development groups in the world toward the target of an EUV light source that will meet the requirements of lithography in radiation power, wavelength, bandwidth, lifetime, etc., with reasonable efficiency and $\operatorname{cost}^{(8)}$. Between these two potential approaches, the discharge produced plasma ${ }^{(9)(10)}$ (DPP) has advantages in high conversion efficiency and low system cost, while having difficulties in debris control and electrode lifetime. It is widely expected that the DPP based light source will be loaded on the first test machine of EUV lithography.

The DPP is powered by pulsed high-current generator. A

\footnotetext{
* Extreme Energy-Density Research Institute, Nagaoka University of Technology

1603-1, Kamitomioka, Nagaoka 940-2188

** Nichicon Corporation

2-3-1, Yagura, Kusatsu 525-0053
}

fast-rising pulsed high-voltage initiates an uniform discharge in low-pressure gas, which is followed by an intense pulsed current. The self-magnetic force of the discharge current compresses the discharge plasma in radial direction resulting in a high-density, high-temperature plasma from which short-wavelength radiation is emitted. By optimizing the gas species, the gas pressure, and the discharge parameters, the radiation power with wavelength near $13.5 \mathrm{~nm}$ can be maximized.

Various studies have been carried out in order to investigate the process of DPP and to improve the power and quality of EUV radiation. As a result, the record of in-band radiation power has been increasing continuously in the last few years, and many research reports on debris and lifetime issues have been published $^{(11)}$. On the other hand, it is noticed that very few publications have been focused on the pulsed high-current generator and its coupling with the discharge load, compared with those focused on the EUV plasmas. Also, in most of the previous works, the deposited energy in the plasma has been estimated by using the difference in the voltage on the final stage capacitor before and after discharge. It includes the loss in the magnetic core and that caused by the internal resistance of the capacitor. In addition, the time dependence of energy deposition was not clear.

We have been developing repetitive pulsed high-current generator $^{(12)}$ for applications in EUV generation. Our interest is on the generator behavior and its performance with DPP load. We have studied the characteristics of the high-current generator with emphasis on the electrical efficiency and the impedance matching with the discharge load, where special efforts have been made in voltage and current diagnostics.

In this paper, we report the experimental results we have obtained recently on the characterization of pulsed high-current 


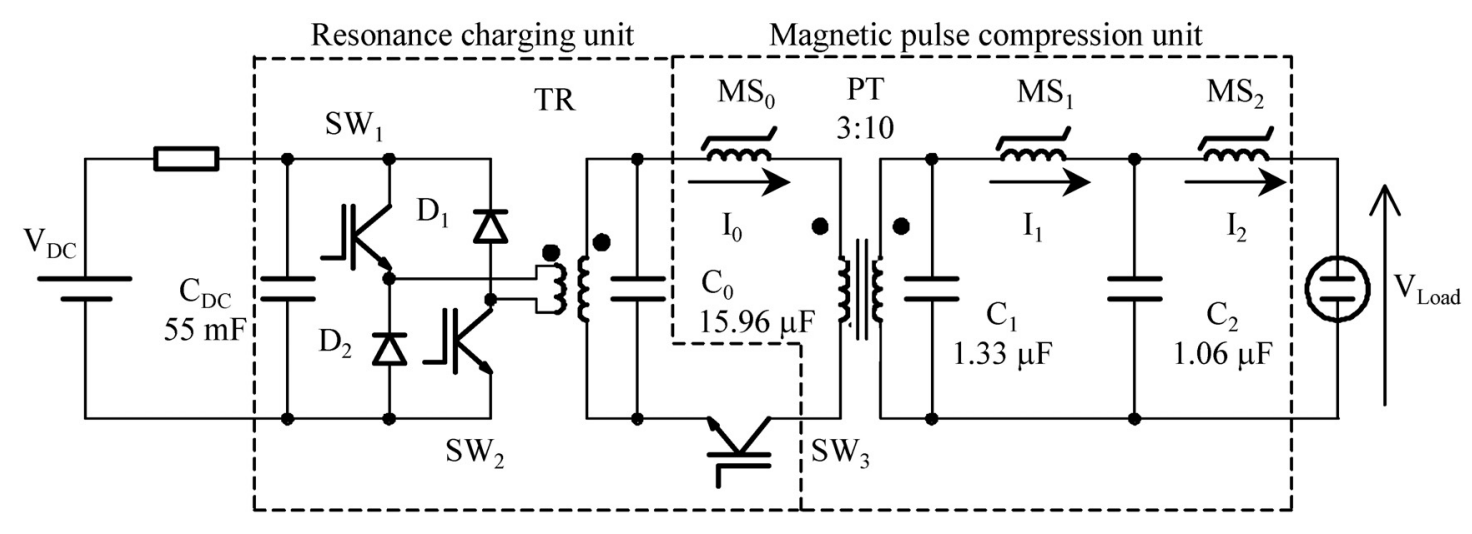

Fig. 1. Schematic diagram of pulsed high-current generator

generator when it is used to drive a capillary discharge load. The study is concentrated on the electrical performance of the generator and its response to the dynamic behavior of the plasma which is monitored by high-speed camera and photo diodes. The time-dependent energy deposition into the discharge plasma is calculated by using the waveforms of the load voltage and current.

\section{Experimental Setup}

The diagram of the pulsed high-current generator we have studied is shown in Fig.1. It basically consists of two units, the resonant charging unit which is used to charge the capacitor $\mathrm{C}_{0}$, and the magnetic pulse compression unit which compresses the pulsed output of $\mathrm{C}_{0}$.

The resonant charging unit operates in the following scheme. The capacitor $C_{D C}$ is first charged by DC power supply of $V_{D C}$. After this charging is completed, IGBT switches $\mathrm{SW}_{1}$ and $\mathrm{SW}_{2}$ are turned on simultaneously. A current flows via $\mathrm{C}_{\mathrm{DC}}-\mathrm{SW}_{1}-\mathrm{TR}-$ $\mathrm{SW}_{2}$ and $\mathrm{C}_{0}$ is charged by resonant current flowing in the secondary winding of the transformer $\mathrm{TR}$. Since $\mathrm{SW}_{1}$ and $\mathrm{SW}_{2}$ are connected in series, their switching voltage is only half of the operating voltage.

The magnetic pulse compression unit operates as follows. When $\mathrm{C}_{0}$ charging is completed, the main IGBT switch $\mathrm{SW}_{3}$ is turned on and the voltage is applied on the magnetic switch $\mathrm{MS}_{0}$. $\mathrm{MS}_{0}$, which saturates in a very short time, is used to decrease the switching loss in $\mathrm{SW}_{3}$. The voltage is then multiplied by pulse transformer PT and is applied on $\mathrm{C}_{1}$. Afterwards, the short pulse compressed by $\mathrm{MS}_{1}$ and $\mathrm{MS}_{2}$ is delivered to the load. More details about the pulsed high-current generator are described in Ref. (11)

The magnetic pulse compression unit is placed in a tank filled with transformer oil. However, the input voltage $\left(\mathrm{V}_{\mathrm{C} 0}\right)$ and current $\left(\mathrm{I}_{0}\right)$, as well as the output voltage $\left(\mathrm{V}_{\text {load }}\right)$ and current $\left(\mathrm{I}_{2}\right)$ are closely monitored by using high-voltage probes and Rogowski coils.

Shown in Fig. 2 are (a) the photograph of the capillary electrode taken by a digital camera and (b) its cross-sectional view, respectively. Anode and cathode are made of stainless steel and a $\phi 5 \mathrm{~mm}$-hole on an alumina plate with $10 \mathrm{~mm}$ in thickness is used as the capillary. The discharge load is placed in a vacuum chamber, which is evacuated by a turbo-molecular pump to $5 \times$ $10^{-3} \mathrm{~Pa}$. After that, the xenon gas with $50 \mathrm{sccm}$ of gas flow rate is introduced from anode side of the capillary.

The behavior of the discharge plasma is monitored by using a high-speed camera (UltraNAC FS501), photodiodes in visible

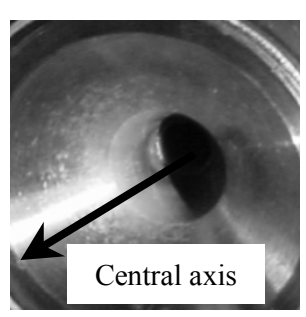

(a)

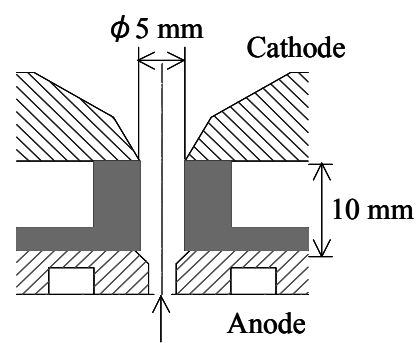

Xenon gas feed

(b)
Fig. 2. Photograph (a) and cross-sectional view (b) of the capillary electrode

band ( AEPX65), and a combination of photodiode (SXUV-HS5) with $\mathrm{Zr}$ filter and $\mathrm{Mo} / \mathrm{Si}$ multilayer mirror for the band of $\pm 2 \%$ at $13.5 \mathrm{~nm}$. The purpose of these observations is to determine the timing of pinch and EUV radiation.

\section{Results and Discussions}

Figure 3 shows the voltage and current waveforms obtained at the capillary discharge load under experimental condition of $\mathrm{C}_{0}=$ $1.08 \mathrm{kV}$. From Fig. 3, the behavior of the last stage of magnetic pulse compression can be clearly seen. After the saturation of $\mathrm{MS}_{1}$, capacitor $\mathrm{C}_{2}$ is charged in approximately $1 \mu \mathrm{s}$. During this charging, in ideal situation, the magnetic switch $\mathrm{MS}_{2}$ should eventually saturate so that, when $\mathrm{V}_{\mathrm{C} 2}$ reaches its maximum, the output should be initiated in the loop of $\mathrm{C}_{2}-\mathrm{MS}_{2}-$ Load. However, since the capillary discharge load has an initially high impedance which does not allow leakage current to saturate $\mathrm{MS}_{2}$, the charging of $\mathrm{C}_{2}$ is accomplished without $\mathrm{MS}_{2}$ saturation and, therefore, without output discharge current ((a) in Fig. 3). On the other hand, as no current is flowing through $\mathrm{MS}_{2}$, there is no significant voltage drop across it and the capillary gap sees the same voltage as $\mathrm{C}_{2}$. At a certain time (the boundary between (a) and (b) in Fig. 3), a breakdown occurs in the capillary. The sudden drop of the load impedance shifts the voltage from the capillary to $\mathrm{MS}_{2}$, initiating the magnetic flux accumulation in its core. After a time of $\sim 700 \mathrm{~ns}$, time required for $\mathrm{MS}_{2}$ saturation ((b) in Fig. 3), magnetic switch $\mathrm{MS}_{2}$ turns on, followed by a large current to the load. Meanwhile, it is interesting to note that the initial discharge before $\mathrm{MS}_{2}$ saturation may have played a role of preionization in the capillary before the main discharge ${ }^{(7)}$.

Figure 4 shows the waveforms of load current, output of visible-light photodiode and visible light images taken by the 


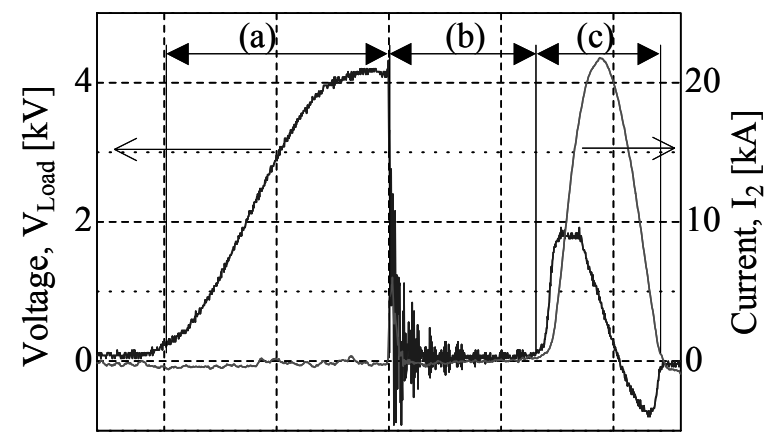

Time $500 \mathrm{~ns} / \mathrm{div}$

Fig. 3. Typical waveforms of load voltage and current

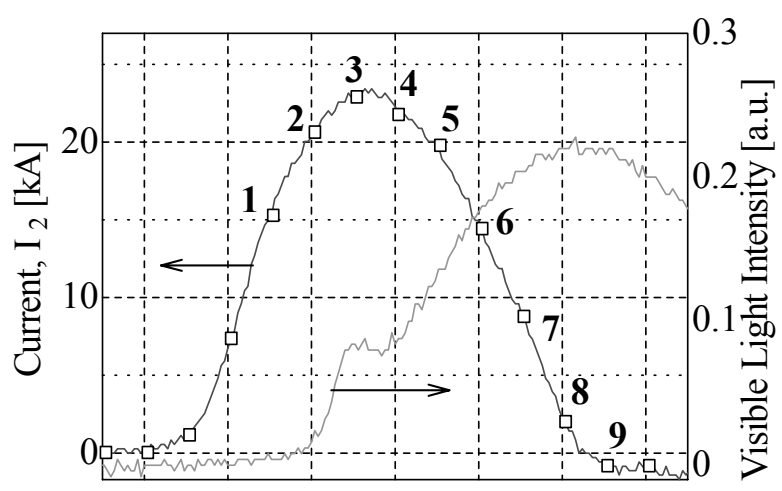

Time $100 \mathrm{~ns} / \mathrm{div}$

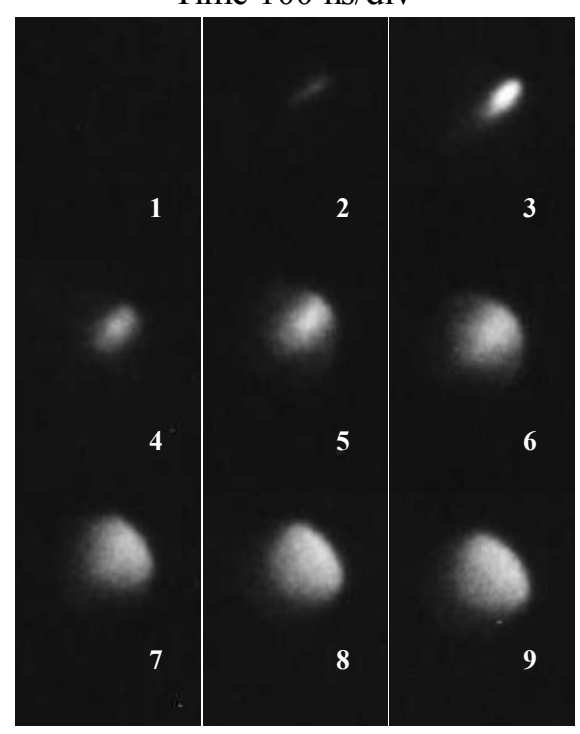

Fig. 4. Waveforms of discharge current and visible light emission, and time-resolved visible light images of the capillary discharge obtained by high-speed framing camera, with exposure time of $10 \mathrm{~ns}$ and frame interval of $40 \mathrm{~ns}$

high-speed camera from the same angle as in Fig. 2 under experimental condition of xenon flow rate of $50 \mathrm{sccm}$. The number under each photograph shows the timing of the exposure corresponding to the numbers marked on the current waveform. The current pulse has a peak value of $\sim 23.2 \mathrm{kA}$ and a full-width of $\sim 500 \mathrm{~ns}$. It can be seen that the signal of visible light has two peaks. The first peak appears around the time of maximum discharge current and the second peak appears after the current finished flowing. The reason can be explained by the visible

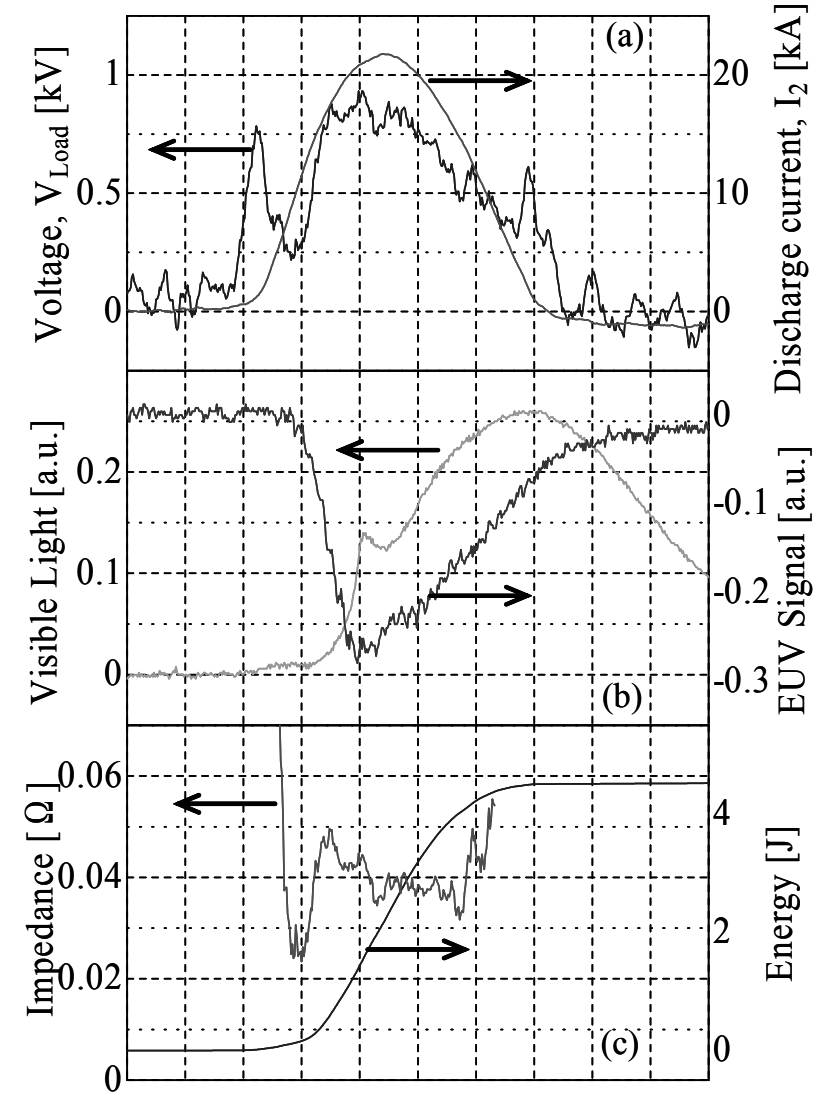

Time $100 \mathrm{~ns} / \mathrm{div}$

Fig. 5. (a) Waveforms of load voltage and current, (b) Waveforms of visible and EUV light emission, (c) Load impedance and energy

light images. During the rising of the discharge current, there is no significant visible light emission from the plasma until the pinch occurs on the center axis. The small and bright pinch plasma corresponds to the first photodiode peak. After that, during the relaxation of the plasma, it expands out of the capillary and forms a stronger emission source due to its large surface area.

Figure 5 shows (a) the waveforms of voltage across the capillary and current, (b) waveforms of visible and EUV light emission obtained by photodiodes, and (c) load impedance and energy obtained from the waveforms of (a) at xenon flow rate of $50 \mathrm{sccm}$. It is noted that the voltage waveform shown in Fig. 5(a) is different with that shown in Fig. 3, which is caused by the inductance correction. There is an unavoidable distance between the measurement point of load voltage and the capillary. Although the inductance $\left(\mathrm{L}_{\mathrm{D}}\right)$ caused by this distance is very small (on the order of $\mathrm{nH}$ ), its effect is enhanced by the large current rising-rate (on the order of $10^{11} \mathrm{~A} / \mathrm{S}$ ). The value of the inductance is approximately $10 \mathrm{nH}$, which is determined by measuring the voltage and current when the capillary is shorted by a metal rod. Therefore, the voltage waveform shown in Fig. 5(a) is obtained by subtracting $\mathrm{L}_{\mathrm{D}} \mathrm{di} / \mathrm{dt}$ from that shown in Fig. 3 and neglecting the resistive loss in the conductors. However, it has to be noted that, although the inductance correction described above is carried out with enough care, the dynamic variation of the load inductance is not taken into account. Therefore, the load impedance obtained (Fig.5(c)) attributes to both capillary resistance and capillary internal inductance. 
Table 1. Energy efficiency

\begin{tabular}{c|c|c|c}
\hline & $\mathrm{C}_{0}$ & $\mathrm{C}_{2}$ & Load \\
\hline Capacitance $[\mu \mathrm{F}]$ & 15.96 & 1.06 & - \\
\hline Voltage $[\mathrm{kV}]$ & 1.10 & 3.96 & - \\
\hline Energy $[\mathrm{J}]$ & 9.3 & 8.3 & 4.5 \\
\hline Efficiency $[\%]$ & 100 & 86.0 & 54.2 \\
\hline
\end{tabular}

The measurement setup (bandwidth is $200 \mathrm{MHz}$ ) for EUV emission consists of a zirconium filter (Luxel Corp.), a $\mathrm{Mo} / \mathrm{Si}$ multilayer mirror and an un-coated silicon photodiode (IRD Inc., SXUV-HS5). This system has measurable band of $\pm 2 \%$ at 13.5 nm. From Fig. 5(b), it is seen that maximum EUV radiation occurs around the time of peak discharge current which, according to Fig. 4, corresponds to the timing of the pinch. In fact, the maximum EUV radiation is obtained after optimization by adjusting the charging voltage and the gas pressure.

The load energy shown in Fig. 5(c) is obtained by integrating the product of voltage and current of Fig. 5(a). From this result, the total deposited energy per pulse is obtained to be $\sim 4.5 \mathrm{~J}$. However, the energy deposition up to the timing of the pinch (peak of EUV radiation) is only $1.4 \mathrm{~J}$. The impedance is calculated from the ratio of voltage and current. No significant impedance variation was observed and the impedance at peak current was obtained to be $\sim 40 \mathrm{~m} \Omega$. Since Ldi/dt $=0$ at the instant of peak current, the load impedance obtained at this moment can be considered as the instantaneous capillary resistance. On the other hand, the output impedance of the generator is calculated to be approximately $150 \mathrm{~m} \Omega$. Impedance mismatching may have prevented efficient energy deposition.

The total energy efficiency is summarized in Table I. We have found out that, from the electric point of view, about half of the circuit energy is coupled into the plasma, which is interesting to be noticed and is an important starting point regarding further optimization of the generator.

\section{Summary}

The results obtained above have given us information about the performance of the high-current generator with a dynamic load of capillary discharge. The circuit behavior of the last stage of the magnetic pulse compression has been clarified and the time-resolved movement of the discharge plasma has been observed. The electrical response of the output waveforms has been analyzed by using diagnostic results and the overall energy efficiency and impedance during the discharge have been obtained from the analytic results. As a result, the total energy deposited in the plasma per pulse was obtained to be $\sim 4.5 \mathrm{~J}$, among which only $\sim 1.4 \mathrm{~J}$ was deposited before the pinch occurred.

(Manuscript received June 12, 2006 , revised Nov. 16, 2006)

\section{References}

(1) K. Murakami, T. Oshino, H. Kinoshita, T. Watanabe, M. Niibe, M. Ito, H. Oizumi, and H. Yamanashi : Jpn. J. Appl. Phys., Vol.37, pp.6750-6755 (1998)

(2) R. H. Moyer, H. Shields, A. Martos, S. W. Fornaca, R. J. St. Pierre, and M. B. Peach : Proc. of SPIE, Vol.4343, p.249 (2001)

(3) W. N. Partlo, I. V. Fomenkov, R. M. Ness, R. I. Oliver, S. T. Melnychuk, and J. E. Rauch : "Progress Toward use of a Dense Plasma Focus as a Light Source for Production EUV Lithography", Proc. of SPIE, Vol.4343 (2001)

(4) International Technology Roadmap for Semiconductor Web Site, update 2004, http://public.itrs.net/

(5) Y. Tomie : "Present Status of Laser Produced Plasma EUV Sources Development”, J. Plasma Fusion Res., Vol.79, No.3, pp.234-239 (2003) (in Japanese)

(6) A. Endo : "High Average Power Laser Produced Plasma EUV Light Sources", J. Plasma Fusion Res., Vol.79, No.3, pp.240-244 (2003) (in Japanese)

(7) E. Hotta : "Present Status of Discharge Produced Plasma Light Sources Development", J. Plasma Fusion Res., Vol.79, No.3, pp.245-251 (2003) (in Japanese)

(8) Y. Watanabe, K. Ota, and H. Franken : Presented at International SEMATECH EUV source Workshop, Antwerp, Belguim (2003)

(9) Y. Teramoto, H. Sato, K. Bessho, T. Shirai, D. Yamatani, T. Takemura, T. Yokota, K. C. Paul, K. Kabuki, K. Miyauchi, M. Ikeuchi, K. Okubo, K. Hotta, M. Yoshioka, and K. Toyoda : "High repetition rate MPC generator-driven capillary Z-pinch EUV source”, Proc. of SPIE, Vol.5374 (2004)

(10) I. V. Fomenkov, N. Böwering, C. L. Retting, S.T. Melnychuk, I. R. Oliver, J. R. Hoffman, O. V. Khodykin, R. M. Ness, and W. N. Partlo : "EUV discharge light source based on a dense plasma focus operated with positive and negative polarity", J. Phys. D: Appl. Phys., Vol.37, p.3266 (2004)

(11) I. V. Fomenkov, W. N. Partlo, R. M. Ness, I. R. Oliver, S. T. Melnychuk, O. V. Khodykin, and N. R. Böwering: "Optimization of a Dense Plasma Focus Device as a Light Source for EUV Lithography", Proc. of SPIE., Vol.4688, pp.634 (2002)

(12) N. Oshima, Y. Kubota, T. Yokoo, K. Shimada, A. Tokuchi, W. Jiang, and K. Yatsui : IEEJ Trans. FM, Vol.125, No.1, p.25 (2005)

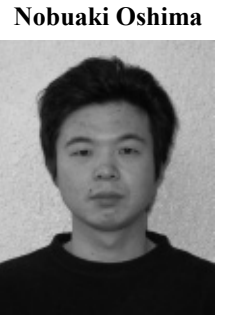

(Student Member) was born in Gunma, Japan in 1979. He received the associate degree in Department of Information and Computer Engineering from Gunma National College of Technology, Japan, in 2000. He received the faculty degree in Department of Electrical Engineering in 2002 and the M. S. degree in Electrical Engineering in 2004, from Nagaoka University of Technology, Japan. His research interests include the pulse power technology and development extreme ultraviolet source.

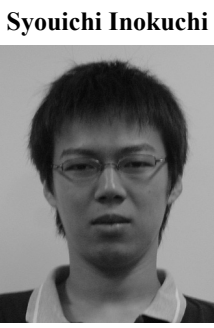

(Student Member) was born in Fukuoka, Japan, on September 17, 1984. He received an academic degree in Nagaoka University of Technology in 2005, and is presently a graduate student at Extreme Energy-Density Research Institute (EDI), Nagaoka University of Technology. He has studied on extreme ultraviolet (EUV) and pulsed power supply.

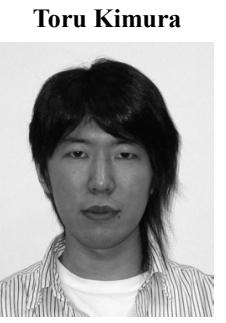

(Non-member) was born in Niigata, Japan, on October 28, 1983. He received an academic degree in Nagaoka University of Technology in 2004, and is presently a graduate student at Extreme Energy-Density Research Institute (EDI), Nagaoka University of Technology. He has studied on extreme ultraviolet (EUV) and pulsed power supply.

Weihua Jiang

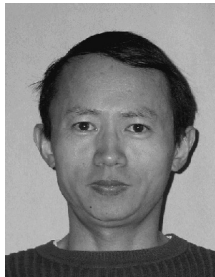

(Member) was born in Beijing, China, in 1962. He received the B.S. degree in engineering physics from the University of Defense Technology, Changsha, China, in 1982 and the M. S. degree in plasma physics from the Institute of Atomic Energy, Beijing, in 1985. He received the $\mathrm{Ph}$. D. degree in electrical engineering in 1991 from Nagaoka University of Technology, Japan. $\mathrm{He}$ is currently an associate professor of Electrical Engineering at Nagaoka University of Technology. His research interests are applications of pulsed particle beams with emphasis on material development and high-power microwave generation. 
Kiyoshi Yatsui (Member) was born in Japan on November 3, 1939. He received the B.S. degree in electrical engineering from Toyama University, Japan, in 1962 and the M.S. and Ph.D. degrees in electrical engineering from Osaka University, Japan, in 1964 and 1968, respectively. From 1968 to 1978, he was with the Faculty of Engineering Science, Osaka University, as a Research Associate in the area of plasma physics. In 1978, he joined Nagaoka University of Technology, Japan, as an Associate Professor. There he started research and development of pulsed power technology and its applications, particularly associated with nuclear fusion, materials science, and gaseous lasers. From 1979 to 1980, he worked as a Visiting Associate Professor at Cornell University. In 1985, he became a Professor in the Department of Electrical Engineering and Director of the Laboratory of Beam Technology. In 1999, he has been the Professor and the Director of Extreme Energy-Density Research Institute, Nagaoka University of Technology. Dr. Yatsui was awarded by the Outstanding Achievement Award of the Institute of Electrical Engineers of Japan in 1993.
Akira Tokuchi (Non-member) received his Master Degree from

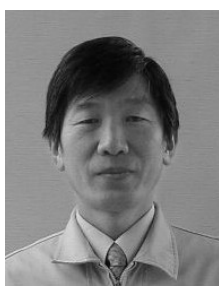

Nagaoka University of Technology in 1983. His thesis was on generation and focusing of medium mass ion beams. He joined Nichicon Corporation in the same year, where he is now at the position section chief of pulsed-power equipments. His research interest is on pulsed high-voltage supply, DC high-voltage supply, and applications on induction accelerators. 